\title{
Las perspectivas de las y los adolescentes sobre la práctica sexual en comunidades de Chiapas, México
}

The perspectives of adolescents on sexual practice in communities in Chiapas, Mexico

Perspectivas dos adolescentes sobre a prática sexual em comunidades de Chiapas, México

Oscar Cruz Pérez Universidad de Ciencias y Artes de Chiapas, Chiapas, México oscar.cruz@unicach.mx https://orcid.org/0000-0003-2452-2834

Hildebertha Esteban Silvestre Instituto de Intervención Psicosocial, México hildeestebans@hotmail.com https://orcid.org/0000-0002-7736-9717

Germán Alejandro García Lara Universidad de Ciencias y Artes de Chiapas, Chiapas, México german.garcia@unicach.mx https://orcid.org/0000-0002-4075-4988

Jesús Ocaña Zúñiga Universidad de Ciencias y Artes de Chiapas, México jesus.ocana@unicach.mx https://orcid.org/0000-0003-0300-0797

Carlos Eduardo Pérez Jiménez Universidad de Ciencias y Artes de Chiapas, México carlos.perez@unicach.mx https://orcid.org/0000-0001-7051-9017 
Revista Iberoamericana

de las Ciencias Sociales y

Humanísticas

\section{Resumen}

Este artículo es producto de una investigación cualitativa realizada con bachilleres de tres comunidades del municipio de Tzimol, Chiapas, para conocer las perspectivas que tienen las y los adolescentes en el ejercicio de su sexualidad. La adolescencia se caracteriza como un periodo de la vida donde las pulsiones sexuales se intensifican; por tanto, se necesita encontrar formas de canalizarlas y dirigirlas hacia objetos sexuales fuera de las relaciones familiares, que ponen en tensión al adolescente con las normas morales, sociales y culturales. La información empírica es reflexionada a partir de la perspectiva de género reconociendo el contexto social donde se insertan las y los adolescentes, el cual define perspectivas distintas para los hombres y las mujeres. En los mecanismos racionales que utilizan para postergar el tiempo para las experiencias sexuales, ellas y ellos reproducen los estereotipos de género.

Palabras clave: adolescencia, embarazo, perspectiva de género, sexualidad.

\section{Abstract}

This article arises from a qualitative research done with high school graduates from three communities of Tzimol, Chiapas, in order to learn about perspectives that adolescents have about the exercise of their sexuality. Adolescence is characterized as a stage of life where sexual pulsations are intensified, in that way, they need to find ways to channel and direct them towards sexual objects outside family relationships, which put adolescents in tension with moral, social and cultural norms. The empirical information is reflected from the perspective of gender recognizing the social context where adolescents are inserted, which defines different perspectives for men and women. In rational mechanisms they use to postpone time for sexual experiences, boys and girls reproduce gender stereotypes.

Keywords: Adolescence, pregnancy, gender perspective, sexuality. 
Revista Iberoamericana

de las Ciencias Sociales y

Humanísticas

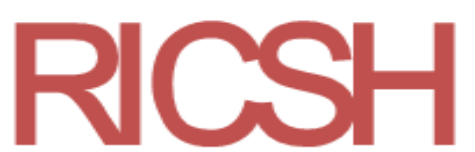

ISSN: $2395-7972$

\section{Resumo}

Este artigo é produto de pesquisa qualitativa realizada com egressos do ensino médio de três comunidades do município de Tzimol, Chiapas, para conhecer as perspectivas que os adolescentes têm no exercício de sua sexualidade. A adolescência é caracterizada como um período da vida em que os impulsos sexuais se intensificam; portanto, é necessário encontrar meios de canalizá-los e direcioná-los para objetos sexuais fora das relações familiares, o que coloca o adolescente em tensão com normas morais, sociais e culturais. As informações empíricas são refletidas na perspectiva de gênero, reconhecendo o contexto social em que os adolescentes estão inseridos, o que define diferentes perspectivas para homens e mulheres. Nos mecanismos racionais que eles usam para adiar o tempo para experiências sexuais, eles e eles reproduzem estereótipos de gênero.

Palavras-chave: adolescência, gravidez, perspectiva de gênero, sexualidade.

Fecha Recepción: Junio 2019

Fecha Aceptación: Diciembre 2019

\section{Introducción}

Definir el concepto adolescencia es problemático, pues la madurez física, los procesos emocionales que se viven y el desarrollo cognitivo que caracterizan a este periodo de desarrollo del ser humano dependen de cada individuo y del contexto donde se ubica. Su inicio y finalización tiene que ver con las características individuales, las condiciones materiales y simbólicas del contexto social, los procesos culturales y las leyes de los

diferentes países (Unicef, 2011). Inclusive, "hacer referencia al comienzo de la pubertad, que podría considerarse una línea de demarcación entre la infancia y la adolescencia, no resuelve el problema” (Unicef, 2011, p. 8). En relación con su finalización, la Secretaría de Salud (2002) sostiene lo siguiente: "La resolución de la adolescencia se da cuando el/la joven ha logrado definir una manera de pensar, sentir y actuar más o menos estable que le permite autonomía personal y adaptación social" (p. 18), características que definen a la adultez.

Esta manera de entender lo adulto por la Secretaría de Salud deja entrever algunas ambigüedades, ya que los conceptos: estable, autonomía personal y adaptación social son difíciles de precisar. La adolescencia se entiende como la etapa del desarrollo que transcurre desde el inicio de la pubertad (aparición de los caracteres sexuales secundarios a 
Revista Iberoamericana

de las Ciencias Sociales y

Humanísticas

ISSN: 2395 - 7972

raíz de cambios hormonales) hasta la finalización del crecimiento biológico y del desarrollo psicológico y social del individuo (Eddy-Ives, 2014), que también nos deja la indefinición de lo que constituye el desarrollo psicológico y social para esta etapa.

Aun así, los diferentes autores e investigadores concuerdan en que la adolescencia inicia con la pubertad, donde se experimenta una repentina transformación en el cuerpo biológico y un efecto en la subjetividad del adolescente. Las transformaciones en el cuerpo y el desarrollo de los órganos sexuales y reproductores dan inicio a la maduración sexual, que se distingue por la intensificación de las pulsiones sexuales que necesitan encontrar formas de canalización y dirigirse a objetos sexuales fuera de las relaciones familiares. Esto obliga al sujeto a abandonar su identidad infantil.

La aparición de la menstruación en las mujeres y la presencia del semen en los hombres imponen el rol genital (un papel por desempeñar) en la reproducción de la especie, pero además exige la construcción de una nueva identidad. Para Aberasturi y Knobel (1987) el adolescente tiene la necesidad de elaborar el duelo por el cuerpo infantil perdido y también por el sexo opuesto perdido (la madre o el padre). Estos cambios implican la construcción de una representación del propio cuerpo, de lo que puede o no hacer con él. Este cuerpo que ha dejado de ser infantil y se ha convertido en cuerpo adulto crea ciertas exigencias en lo estético y en lo sexual convirtiéndose en objeto de satisfacción y de admiración en el púber.

La renuncia al cuerpo infantil le exige una lenta y dolorosa labor de duelo que incluye al cuerpo, la mente y las relaciones de objetos infantiles. Al respecto, Freud (1916) sostiene que en la época de la pubertad, cuando la pulsión sexual plantea sus exigencias por primera vez con toda su fuerza, las figuras parentales son retomadas e investidas de nuevo libidinalmente. Desde este momento, el individuo humano tiene que consagrarse a la gran tarea de abandonar a su padre y madre como objetos de amor incestuoso y dirigir la libido hacia otras personas fuera del ámbito familiar. Este cuerpo marcado por el deseo de la madre y por la frustración constituye la dinámica de su deseo que se transforma en formas de sentir, actuar, desear y en todas las manifestaciones humanas.

El estudio actual de la adolescencia por parte de distintas disciplinas tiene ante sí la consigna de brindar posibilidades para comprender mejor los procesos de relación entre adolescentes y adultos, los desafíos de su salud sexual y reproductiva, su ingreso al mercado laboral, las situaciones de riesgo psicosocial, de marginación y de exclusión a que 


\section{Revista Iberoamericana de las Ciencias Sociales y Humanísticas}

ISSN: 2395 - 7972

se enfrentan, entre otros (Lozano Vicente, 2014). Si bien "la adolescencia es una etapa de vida con grandes oportunidades también es altamente vulnerable, pues las decisiones sobre la conducta sexual y reproductiva que se tomen en este momento son trascendentes para el desarrollo futuro de los individuos, de sus hijos o hijas y de sus parejas" (Díaz-Sánchez, 2003, p. 23). Esto considerando que diversas investigaciones han encontrado que en el periodo de la adolescencia "resulta muy fácil incurrir en relaciones sexuales íntimas, que pueden culminar con un embarazo, más aún si no utilizan algún método anticonceptivo" (Soto-Martínez, Franco-Bonal, Franco-Bonal, Silva-Valido y Velázquez-Zúñiga, 2003, párr. 24.).

Según Romero (2016), la Organización para la Cooperación y el Desarrollo Económico (OCDE) reporta que México ocupa el primer lugar de embarazos adolescentes con cerca 400000 mujeres menores de 19 años que son madres. Esta condición hace que el embarazo adolescente en México sea considerado un problema de salud pública. El Consejo Nacional de Población estimó que en el año 2014 en México una de cada cinco personas tenía entre 10 y 19 años de edad, y Chiapas fue el estado con mayor porcentaje de adolescentes con $21.8 \%$.

La tasa de fecundidad nacional para el grupo de 15 a 19 años en 2009 fue de 69.5, en tanto que Chiapas ocupó el tercer lugar nacional con 89.1. Estos datos pueden explicarse debido a que Chiapas cuenta con el porcentaje más alto de adolescentes indígenas (29 \%) y la población de habla indígena reporta mayores tasas de fecundidad que la población no indígena (Estrategia Nacional para la Prevención del Embarazo en Adolescentes [Enapea], 28 de marzo de 2017).

Según el Inegi (2014), el embarazo adolescente como problemática de salud pública registra altos índices de muerte materna, mayor probabilidad de muerte prenatal o perinatal, bajo peso al nacer y riesgos para la salud de la madre adolescente, como obesidad, hipertensión, preclamsia, anemia e infecciones de transmisión sexual. Estas complicaciones asociadas al embarazo adolescente no son necesariamente causadas por la edad (15 a 19 años), sino por las condiciones previas al embarazo, como la mala nutrición, bajo peso y talla en la adolescente, ausencia y/o mala calidad de control prenatal, así como condiciones socioeconómicas precarias (Díaz, Sanhueza y Yaksic, 2002; Reyes y González Almontes, 2014). Las niñas y adolescentes más pobres y con una educación deficiente son quienes 
Revista Iberoamericana

de las Ciencias Sociales y

Humanísticas

ISSN: $2395-7972$

tienen más riesgo de un embarazo a esta edad (Fondo de Población de las Naciones Unidas [UNFPA], 2013).

Los nacimientos de mujeres adolescentes tienen mayor prevalencia en municipios con altos grados de marginación. Chiapas es uno de los estados con menor PIB per cápita del país con -1.1, lo que contrasta con otras entidades como Ciudad de México, que registra 4.6 y tiene la menor proporción de población adolescente del país (Enapea, 28 de marzo de 2017; Inegi, 2016).

El embarazo adolescente repercute en la deserción escolar, rechazo social, conflictos familiares y escasos ingresos, lo que implica falta de seguridad social y menores oportunidades de acceso a servicios de salud (Secretaría de Educación Pública [SEP], 2012). Se ha comprobado que aun en condiciones de pobreza, la existencia de mayores oportunidades para seguir en la escuela y acceder a un trabajo remunerado son determinantes para retrasar la primera relación sexual, el matrimonio y el embarazo (Enapea, 28 de marzo de 2017).

La cultura androcéntrica y machista sigue profundamente arraigada en las prácticas cotidianas que caracterizan al estado de Chiapas, en lo particular al municipio de Tzimol, situación que favorece la reproducción de representaciones sociales de las mujeres básicamente como madres y esposas. Los roles tradicionales del ser madre y esposa determinan las posibilidades de desarrollo personal de las mujeres, los cuales provienen desde la enseñanza y el aprendizaje de una posición de subordinación frente a los hombres desde el hogar, como niñas que deben aprender las labores que desempañarán más tarde mediante la reproducción de las prácticas de obediencia y sometimiento (Instituto Nacional de las Mujeres [Inmujeres], 2016a).

Tomando en consideración estas lógicas de pensamiento, en el presente trabajo se analizan las perspectivas sobre el ejercicio de la sexualidad y el embarazo que tienen las y los adolescentes de los centros escolares de nivel bachillerato del municipio de Tzimol, Chiapas. 
Revista Iberoamericana

de las Ciencias Sociales y

Humanísticas

ISSN: $2395-7972$

\section{Espacio social de investigación}

El municipio de Tzimol forma parte de la región XV, Meseta Comiteca Tojolabal integrada por seis municipios más: Comitán de Domínguez, La Independencia, La Trinitaria, Las Margaritas, Las Rosas y Maravilla Tenejapa. Tiene una extensión territorial de $360.71 \mathrm{~km}^{2}$, y ocupa $0.49 \%$ de la superficie del estado. Cuenta con 73 localidades y según el conteo intercensal de 2015 (Inegi, 2015), la población ascendió a 15316 (7431 hombres y 7885 mujeres). El total de personas del municipio constituye $0.29 \%$ de la población total del estado de Chiapas, distribuidas en 73 localidades.

Las coordenadas de la cabecera municipal son las siguientes: $16^{\circ} 11^{\prime} 15^{\prime \prime}$ de latitud norte y $92^{\circ} 11^{\prime} 49^{\prime \prime}$ de longitud oeste, y se ubica a una altitud de 1403 metros sobre el nivel del mar. Concentra a 5112 habitantes; el resto de la población se distribuye en las diferentes localidades: las más grandes son La Mesilla con 2604, la colonia Ochusjob con 1173 y Héroes de Chapultepec con 1015, mientras que las demás comunidades cuentan con menos de 500 habitantes (Inegi, 2010).

En el municipio de Tzimol se registraron $84.72 \%$ hogares con jefaturas masculinas en tanto que los hogares en los que las jefaturas son femeninas corresponde a $15.22 \%$, según datos estimado por COESPO, tomando como base el censo de población y vivienda 2010 del Inegi.

La población que habla alguna lengua indígena es muy poca, ya que solo asciende a 195 personas en todo el municipio, lo que equivale a $0.02 \%$ del total de la población, y se localizan en la comunidad de Paso Hondo y La Nueva Libertad. Estas comunidades, por su lejanía con la zona urbana, carecen de muchos servicios y tienen un alto grado de marginación.

De acuerdo con los resultados del Consejo Nacional de Evaluación de la Política de Desarrollo Social (Coneval) (2010) sobre la medición de la pobreza, en el año 2010, 89 \% de los habitantes de Tzimol se encontraban en pobreza, de los cuales 9249 (55\%) presentaban pobreza moderada y 5717 (34\%) pobreza extrema.

En este mismo año, la condición de rezago educativo afectó a $48.5 \%$ de la población y el porcentaje de personas sin acceso a servicios de salud fue de $26.1 \%$. La carencia por acceso a la seguridad social afectó a 83.6 \% de la población, es decir, 14065 personas. El porcentaje de ciudadanos que reportó habitar en viviendas sin disponibilidad de servicios básicos fue de 59.7 \% (10 039 personas) y la incidencia de la carencia por 
Revista Iberoamericana

de las Ciencias Sociales y

Humanísticas

ISSN: $2395-7972$

acceso a la alimentación fue de $29.9 \%$, es decir 5024 personas que no tienen una alimentación adecuada.

\section{Sujetos de estudio}

La población de esta investigación estuvo constituida por 275 estudiantes del nivel medio superior de tres comunidades: cabecera municipal de Tzimol (que lleva el mismo nombre), La Mesilla y Héroes de Chapultepec, de los cuales 54.18 \% fueron hombres y el $45.82 \%$ mujeres. Sus edades oscilaban entre los 14 y los 19 años. De hecho, 257 (93.46 \%) se ubican en edades que van desde los 15 hasta los 17 años.

Tabla 1. Distribución de la población de estudio por sexo y edad.

\begin{tabular}{|c|c|c|c|c|c|c|c|}
\hline \multirow{2}{*}{ Sexo } & \multicolumn{6}{|c|}{ Edades (años) } & \multirow{2}{*}{$\begin{array}{c}\text { Total } \\
\text { sexo }\end{array}$} \\
\cline { 2 - 7 } & $\mathbf{1 4}$ & $\mathbf{1 5}$ & $\mathbf{1 6}$ & $\mathbf{1 7}$ & $\mathbf{1 8}$ & $\mathbf{1 9}$ & $\mathbf{1 4 9}$ \\
\hline Hombre & 3 & 52 & 41 & 46 & 6 & 1 & $\mathbf{1 4 9}$ \\
\hline Mujer & 2 & 39 & 38 & 41 & 5 & 1 & $\mathbf{1 2 6}$ \\
\hline Total edad & $\mathbf{5}$ & $\mathbf{9 1}$ & $\mathbf{7 9}$ & $\mathbf{8 7}$ & $\mathbf{1 1}$ & $\mathbf{2}$ & $\mathbf{2 7 5}$ \\
\hline
\end{tabular}

Fuente: Elaboración propia.

Esta población constituye el cien por ciento de estudiantes de bachillerato en el municipio, ya que solamente existen tres centros educativos: uno en modalidad de plantel formal con una plantilla de docentes por asignatura o materia y dos con modalidad de educación a distancia, los cuales emplean diversos medios de comunicación para la transmisión de los contenidos reforzados por asesorías grupales e individuales. En esta modalidad a distancia solamente existe un docente por campo de conocimiento para las asesorías.

\section{Proceso metodológico}

El trabajo se sustentó en la metodología cualitativa, mediante la cual se intentan comprender las realidades cotidianas desde el contexto de sus participantes. Esta metodología explora los fenómenos y acontecimiento de la cotidianidad de la vida social para analizarlos y reflexionar sobre ellos y para mostrar su complejidad y la forma en que se imbrican las condiciones personales, culturales y estructurales. A través de esta metodología se analizan las perspectivas que tienen las y los adolescentes sobre el ejercicio 
Revista Iberoamericana

de las Ciencias Sociales y

Humanísticas

ISSN: $2395-7972$

de su sexualidad y el embarazo, lo que implica dar cuenta de sus procesos internos subjetivos, describiendo e interpretando los discursos escritos a preguntas abiertas y tomando en cuenta su realidad.

Lo que verdaderamente caracteriza a los métodos cualitativos es su enfoque y finalidad más que el procedimiento de investigación. Con esta metodología se consigue un acercamiento a los directamente implicados y ver el mundo desde su perspectiva, esto constituye su principal atractivo (Pérez-Serrano, 2001, p. 25).

Desde este posicionamiento metodológico se muestran los procesos internos de los y las adolescentes en relación con la sexualidad y el embarazo, lo que exige comprender su realidad desde su propia mirada como sujetos.

Para la recogida de la información se utilizó un cuestionario autoaplicable con preguntas abiertas que los sujetos respondieron de forma libre. El cuestionario exploró datos de identificación personal, del centro escolar y de las siguientes categorías que se tradujeron en preguntas concretas.

a. Expectativas de edad para iniciar relaciones sexuales.

b. Expectativas de uso del condón.

c. Iniciativa para la sugerencia del uso del condón.

d. Fuentes de apoyo en embarazos no planeados.

e. Expectativas de acción en caso de embarazo no planeado.

f. Expectativa personal de edad para tener un hijo/a.

g. Opinión sobre las mujeres que abandonan la escuela.

h. Opinión sobre los hombres que abandonan la escuela.

El análisis y la sistematización de la información se realizaron bajo ciertas etapas diferenciadas. La primera fue el descubrimiento e identificación de información significativa que permitió el desarrollo de temas sobresalientes; posteriormente se organizaron los contenidos de los temas y se construyeron nuevas categorías para, finalmente, comprender dichos discursos desde los propios sujetos sin desconocer el contexto y analizarlos desde los referentes teóricos, es decir, "comprender los datos en el contexto en que fueron recogidos" (Taylor y Bogdan, 1996, p. 159). 


\section{Resultados}

Para conocer las expectativas que tenían los estudiantes sobre la práctica sexual, se les formuló la pregunta ¿Cuál crees que es la mejor edad para empezar a tener relaciones sexuales? Al respecto, la población de estudio señaló que las mejores edades para iniciar la práctica sexual se ubican entre los 18 y los 25 años, con una sumatoria de $79.27 \%$. Las edades de 20 hasta 22 años son las señaladas por una mayor proporción de la población encuestada (31.27\%), mientras que el rango de 18 a 19 años como edad ideal es compartida por $30.45 \%$ de los estudiantes.

Sin embargo, también encontramos que $39.28 \%$ de los estudiantes opinan que la mejor edad para iniciar una vida sexual activa va desde los 15 hasta los 19 años. Este último dato es significativo debido a que nuestra población de estudio transita entre los 14 y los 19 años de edad; por lo tanto, si para ellos la mejor edad para empezar la práctica sexual se ubica en el rango de 15 años a 19 años, significa que se autorizan a sí mismos para iniciar sus relaciones sexuales. Esto es parte del reconocimiento subjetivo propio de la adolescencia respecto al cuerpo púber y/o adolescente, que entraña nuevas sensaciones y vivencias al ir comprendiendo que se es y se tiene, al mismo tiempo, un cuerpo que da la existencia misma. Es importante hacer notar que esta condición subjetiva coloca a las y los jóvenes en un alto riesgo de embarazos no deseados cuando no tienen acceso a información o acompañamiento pertinente.

Desde las teorías psicoanalíticas, se reconoce a la adolescencia como el momento de emergencia de la libido sexual que acompaña a las transformaciones del cuerpo biológico, entre otros, el crecimiento y desarrollo de los órganos sexuales y reproductores que intensifican las pulsiones sexuales. Esta libido emergente debe encontrar formas de canalización y dirigirse a objetos sexuales fuera de las relaciones familiares (Aberasturi et al., 1987). Retomando a Freud (2016), entendemos que la adolescencia es el momento inaugural donde el individuo humano tiene que consagrarse a la gran tarea de abandonar a la figuras parentales como objetos de amor incestuoso y dirigir la libido hacia otras personas fuera del ámbito familiar, lo cual explica lo inevitable que resulta para las y los adolescentes sentirse atraídos sexualmente por su grupo de pares. Sin embargo, la demanda de satisfacción libidinal adolescente choca con la moral vigente en las comunidades rurales como Tzimol, que niega o esconde la práctica sexual especialmente en las mujeres. 
Revista Iberoamericana

de las Ciencias Sociales y

Humanísticas

ISSN: 2395 - 7972

Otro dato importante es que las mujeres parecen tener expectativas de posponer un poco más esta edad de inicio de la vida sexual en comparación con los hombres, pues un mayor porcentaje de ellas ubican esta edad de inicio entre los 20 y 25 años, mientras que los hombres señalan la edad ideal a partir de los 18 y 19 años. Esto también debe ser reflexionado a partir de reconocer el contexto social que sanciona en mayor medida el ejercicio sexual de las mujeres, de quienes se espera un "buen comportamiento", lo que desde la moral vigente se refiere a la abstinencia sexual hasta llegar al matrimonio, es decir, mantener la virginidad, mientras que en el caso de los hombres sucede lo contrario.

Ante la interrogante de por qué consideran la edad señalada como la más idónea para iniciar la práctica sexual, encontramos que las respuestas son diferentes según sean mujeres u hombres.

\section{Expectativas respecto al ejercicio de la sexualidad en los hombres}

En el caso de los hombres, las categorías construidas fueron las posibilidades de ejercer su libertad y sus derechos, la preocupación por tener un trabajo, la seguridad de sostener una responsabilidad familiar y la expectativa de tener una carrera profesional.

\section{Ejercicio de la libertad y el derecho}

Para los hombres la edad del inicio de la práctica sexual después de los 18 años se relaciona con la adquisición de los derechos que implica alcanzar la mayoría de edad, que legalmente otorga autonomía, capacidad de decisión y con ello el derecho a formar una familia. Antes de ser mayor de edad, están bajo la tutela de los padres, lo cual es visto como el principal obstáculo para hacer lo que quieren de forma independiente.

Esto también sugiere que tener relaciones sexuales está fuertemente asociado a la responsabilidad que esto demanda, sinónimo de embarazo y una relación formal de pareja. Es decir, los jóvenes son conscientes de los riesgos que subyacen en la práctica sexual y apelan a su derecho legal para ser libres de tomar tales decisiones, lo cual pueden hacer cuando son mayores de edad. Entre los discursos destacan las siguientes ideas:

- Porque ya tienes mayoría de edad y puedes ser libre.

- Porque en esas edades podemos conocer nuestros derechos.

- Porque ya somos mayores de edad.

- Porque ya me puedo independizar y ser responsable. 
Revista Iberoamericana

de las Ciencias Sociales y

Humanísticas

ISSN: $2395-7972$

- Porque ya eres mayor de edad y ya debes hacerte responsable de tus actos.

- Con esa edad puedes decidir con tu vida [sic].

\section{La preocupación por tener un trabajo}

La mayoría de edad para los jóvenes como la ideal para empezar a tener una vida sexual activa también está vinculada con la condición de tener un trabajo, como la opción para afrontar las consecuencias económicas que eso demanda al no tener ya la tutela de los padres. Es interesante observar que en este discurso se asocia la edad con la capacidad para trabajar, con estar preparado para buscar un empleo y con tener un ingreso económico y el derecho o libertad para tener relaciones sexuales.

- Porque ya podemos tener trabajo.

- Porque ya podemos empezar a trabajar.

- Porque ya trabajo.

- Porque ya tienes trabajo y estás preparado para la vida.

- Porque en ocasiones ya tienen un trabajo.

- Ya tendría un trabajo.

- Porque ya podemos tener un trabajo y hacernos cargo.

- Porque ya puedo comenzar a buscar empleo y tener dinero.

\section{La responsabilidad familiar}

El tercer elemento en las razones que los jóvenes aducen para iniciar su vida sexual entre los 18 y 19 años es la responsabilidad para hacerse cargo de mantener a su familia. Esto significa que tener relaciones sexuales se vincula directamente con tener hijos y formar una familia, ante lo cual la responsabilidad aparece como un valor social con un papel esencial en su identidad como varones, puesto que, como lo veremos adelante, este valor aparece de forma muy diferente para las mujeres.

Esto nos lleva a preguntarnos cuál es el significado de la prevención del embarazo y qué cabida tiene entre los valores de estos jóvenes; a partir de ello, tendrían que revisarse los programas de prevención del embarazo no deseado que se enfocan en la toma de decisiones de las y los jóvenes como sujetos individuales, al margen de sus valores familiares y culturales. Como puede entenderse con estas expresiones de los jóvenes, tener relaciones sexuales es sinónimo de derechos, de libertad y de responsabilidad; sin embargo, 
Revista Iberoamericana

de las Ciencias Sociales y

Humanísticas

ISSN: $2395-7972$

estos principios son difícilmente posibles o reales en la vida cotidiana para la mayoría de ellos, quienes al mismo tiempo - aun sin ser independientes de los padres, sin tener un trabajo o sin haber concluido la carrera - ya ejercen su sexualidad de manera activa.

- Porque así uno no está tan joven y sabe cómo mantener la familia [sic].

- Porque tal vez ya tiene uno trabajo para mantener la familia [sic].

- Porque tienes mejor posibilidad de mantener a los hijos.

- Porque tienes la edad para mantener tu familia [sic].

- Porque tienes la suficiente responsabilidad para poder ser padres.

- Porque ya puedes trabajar o mantener a tu familia.

- Porque es una obligación mantener la familia.

- Pues para ser responsable con la familia [sic].

- Porque uno ya está un poco más maduro para saber tener una familia.

- Porque es la edad que uno ya puede mantener a la pareja.

- Porque si quedan embarazadas su novia ya tiene madures para hacerse cargo del bebé $[s i c]$.

\section{La expectativa de una carrera profesional}

En menor medida se señala como razón para posponer el inicio de las relaciones sexuales a edades avanzadas el concluir una carrera profesional. Esto va de la mano con la condición de tener una mejor oportunidad de trabajo que les permitiría afrontar su responsabilidad como jefes de una familia.

- Porque ya puedes tener una carrera.

- Porque ya terminaste tu carrera.

- Porque ya podemos haber terminado de estudiar [sic].

- Porque algunos piensan tener una carrera y si tienes un hijo no lo puedes hacer.

- Porque ya sacaste la carrera y eres mayor de edad.

- Para terminar los estudios.

- Porque terminaron los estudios.

- Para terminar los estudios. 
Revista Iberoamericana

de las Ciencias Sociales y

Humanísticas

ISSN: $2395-7972$

El valor más fuertemente arraigado y que parece ser el eje en el discurso de estos jóvenes es el de la responsabilidad familiar, la cual esperan cumplir mediante el trabajo. Esto se vincula de manera directa con la libertad para tomar sus propias decisiones y con ello el derecho a tener una vida sexual activa.

Estos datos dan cuenta de la carga cultural que reproducen en su discurso estos hombres jóvenes: en primer lugar, la demanda de sus derechos, esto es, de ser libres para tomar decisiones, lo cual culturalmente es propio del género masculino; asimismo, ser dueños de la palabra, del espacio público, del poder, del control y del dominio sobre los otros. En segundo lugar, la asunción del rol de proveedores, responsables de sostener económicamente a la familia, lo que les impone la necesidad de trabajar de cualquier manera o de estudiar para conseguir un trabajo mejor remunerado.

\section{Expectativas respecto al ejercicio de la sexualidad en las mujeres}

En el caso de las mujeres se construyeron cuatro categorías similares a la de los hombres, aunque expresan significados diferentes: tener una carrera profesional, la preocupación por los cuidados de un embarazo, la capacidad de ser responsables y el desarrollo de habilidades cognitivas.

\section{Tener una carrera profesional}

Esta categoría reúne las respuestas de mayor frecuencia entre las jóvenes como razón para posponer su vida sexual activa después de los 18 años, que es la condición de tener una carrera profesional, sinónimo de estar preparadas para hacerse cargo de las consecuencias naturales de las relaciones sexuales (tener hijos/as).

La aparición de esta categoría como la primera en frecuencia para las mujeres es congruente con el discurso político que impulsa su inclusión a los espacios que tradicional y culturalmente han sido exclusivos de los hombres: el ámbito laboral considerado productivo y remunerable. Las adolescentes reconocen que la formación profesional amplía las oportunidades de las mujeres para trascender del ámbito familiar doméstico, espacio de reproducción de los roles de cuidado que no tienen una remuneración económica, ni reconocimiento social o político, además de ser vivido como sinónimo de sumisión y subordinación al género masculino. Terminar una carrera profesional posibilita su inserción al ámbito laboral fuera de su comunidad y de su contexto social y cultural inmediato. En 
Revista Iberoamericana

de las Ciencias Sociales y

Humanísticas

ISSN: 2395 - 7972

este sentido, podemos dilucidar que la primera razón de las mujeres para posponer el inicio de su vida sexual activa es evitar reproducir los roles tradicionales del género femenino.

Al igual que los hombres, las mujeres aspiran a ejercer sus derechos a ser libres y tomar sus propias decisiones, solo que ellas toman en cuenta otros factores como el acceder a la preparación formal y conseguir un empleo para hacer uso de ellos.

- Porque ya tenemos nuestra carrera.

- Porque estás estudiando y cuando termines la carrera después tener hijos [sic].

- Porque ya acabaste el estudio.

- Porque ya tienes una carrera.

- Porque tiene su carrera y puede hacerse cargo.

- Porque ya estamos preparadas.

\section{Preocupación por los cuidados del embarazo}

La preocupación por el embarazo aparece en todas las edades: las más jóvenes enfocadas en evitarlo y en no enfermar, las mayores se preocupan de saber y poder cuidar de sí mismas y de su bebé. Esta intranquilidad por los cuidados propios de un embarazo no aparece en los hombres, pues es totalmente diferente a la responsabilidad que implica mantener a una familia o tener un trabajo. Tal desasosiego apunta directamente al cuerpo de las mujeres que viven el proceso de embarazo y de su producto. Es también congruente con el encargo social de ser las cuidadoras y responsables de la sobrevivencia del bebé que las jóvenes preparatorianas asumen plenamente como una consecuencia de tener relaciones sexuales.

En cuanto a lo no referido por estas jóvenes, podemos observar que no aluden a la corresponsabilidad de su pareja durante este periodo de embarazo, sino que más bien asumen las implicaciones de cuidar a su hijo/a y su hogar.

- Para no salir embarazada y con enfermedades.

- Para poder protegerse y saber lo que puedes hacer cuidándote con protección.

- Porque es mejor quedar embarazada con más edad.

- Porque si llegamos a quedar embarazada ya lo vamos a poder cuidar [sic].

- Porque ya piensas y ya está desarrollado tu cuerpo.

- Para cuidar un embarazo no planeado.

- Porque ya puede uno saber cuidar un bebé. 
- Porque a esa edad ya está uno grandecita para las responsabilidades de un hogar.

\section{Capacidades para ser responsable}

Para las mujeres tener relaciones sexuales es un comportamiento que exige ser capaz de responsabilizarse de todas las consecuencias que implica tener un hijo/a. A diferencia de los hombres, que especifican la responsabilidad de mantener a su mujer $e$ hijos/as (ser portador de los recursos económicos), para las mujeres esta tarea es asumida en mayor magnitud en franco acatamiento de lo establecido culturalmente: cuidar de la familia incluye tanto cuestiones de producción económica (trabajos de traspatio y ventas informales de diversos productos) como emocionales, afectivas y de seguridad. Además, las mujeres en estas comunidades no son solo responsables de sus propios hijos e hijas o de su propio hogar, sino también de sus padres, madres, hermanos, hermanas, suegros y suegras.

- Porque ya son grandes y pueden hacerse responsables.

- Porque somos más responsables.

- Porque tenemos que ser responsables.

- Porque ya es un poco de tener una parte de responsabilidad [sic].

- Porque ya te haces cargo de tus problemas.

- Porque ya estamos más preparados para eso y estamos listos para una realidad como tener hijos.

- Porque ya podemos tomar las mejores decisiones.

- Porque uno ya sabe que obligaciones tiene $[$ sic $]$.

- Porque es cuando estamos más preparadas y enfrentar todas las consecuencias.

\section{Desarrollo de habilidades cognitivas}

La edad es sinónimo de asunción de responsabilidades y obligaciones para las jóvenes, como una condición importante para tener relaciones sexuales. Ser mayores de edad significa para ellas tener la capacidad de tomar decisiones reflexionadas, tener mayor consciencia de los riesgos y consecuencias de sus decisiones y comportamiento. Sostienen que esa edad es la más conveniente para las experiencias sexuales.

Para estas jóvenes, con la edad adquieren también mayores habilidades cognitivas e intelectuales que les permiten enfrentar de mejor manera las consecuencias de las 
Revista Iberoamericana

de las Ciencias Sociales y

Humanísticas

ISSN: $2395-7972$

relaciones sexuales. El pensar y reflexionar sobre las consecuencias son, para ellas, los motivos centrales para tomar decisiones sobre la práctica sexual.

- Porque ya tiene más experiencia.

- Porque ya piensan más.

- Porque ya tenemos en cuenta que consecuencias hay [sic].

- Pues porque ya tienes una idea y pues ya eres responsable [sic].

- Porque pensamos mejor las cosas.

- Porque ya estoy madura y ya sé que en qué riesgo me comprometo.

- Porque a esa edad ya hemos desarrollado más y piensas bien las cosas.

- Porque son mayores y ya pueden pensar bien las cosas.

- Porque es una edad donde se pueden pensar mejor las cosas.

- Porque tal vez ya estamos consciente de nuestras acciones [sic].

Además del incremento de las pulsiones sexuales en las y los adolescentes, se observa una capacidad cognitiva importante para la reflexión en la toma de decisiones. Existe un reconocimiento en las adolescentes que con el incremento de la edad pueden pensar mejor las situaciones de la vida y particularmente su práctica sexual.

\section{Discusión}

Comprender el tema de la sexualidad y la práctica sexual en la población adolescente es complejo y genera muchas dificultades, ya que tiene que ver con las condiciones y situaciones particulares de los sujetos, los proyectos de vida, las competencias para la toma de decisiones y el contexto sociocultural. En este trabajo únicamente presentamos algunas expectativas que tienen las y los adolescentes en relación con la práctica sexual y sobre todo las "razones" que tienen para ejercerla o no dependiendo de sus recursos cognitivos, oportunidades de formación y aprendizajes a partir de su edad. Sin embargo, abona al conocimiento de la realidad de esta población, siguiendo a JuárezBolaños, Parra-Vázquez, Mariaca-Méndez y Díaz-Hernández (2011), quienes —al cuestionarse sobre los estudios que se han realizado con jóvenes rurales e indígenas en México y Latinoamérica- sostienen que en México no solo existen escasas investigaciones con estas poblaciones, por lo que hace falta construir referentes teóricos para definir y entender a la juventud del medio rural e indígena, sino también que se carece de políticas públicas y acciones dirigidas hacia este grupo vulnerabilizado. 
Revista Iberoamericana

de las Ciencias Sociales y

Humanísticas

ISSN: $2395-7972$

En los datos analizados, además, se observa una tendencia a reproducir los estereotipos de género en la población de estudio; este hallazgo concuerda con la información que presenta la SEP (2013) en el texto Embarazo adolescente y madres jóvenes en México. Una visión desde el Promajoven, donde se analiza la situación de las mujeres embarazadas y su impacto en la educación básica.

El trabajo muestra que tanto los hombres y las mujeres se encuentran en situaciones de tensión ante los impulsos sexuales intensos, las normas y valores culturales propios del contexto donde desarrollan su vida cotidiana y los recursos cognitivos que han adquirido en su proceso de desarrollo. Comprender estas tensiones y el sentido que dan a la práctica sexual dependiendo de su condición genérica brinda elementos para configurar algunas de sus necesidades y permite dibujar estrategias posibles de orientación y apoyo pertinentes. Dentro de las limitantes del estudio es importante mencionar que los datos analizados se recolectan través de un cuestionario abierto autoaplicable, que si bien los y las adolescentes tuvieron la oportunidad de escribir sus ideas y pensamientos, reduce la posibilidad de acceso a la subjetividad a profundidad, por lo que es necesario tiempo y disposición de escucha de las narrativas de ellos y de ellas sobre la sexualidad y sus prácticas. En este sentido, los datos que presentamos necesitan enriquecerse con otras miradas y perspectivas para dar cuenta de mejor manera de esta realidad compleja que nos ocupa.

\section{Conclusiones}

Desde la metodología cualitativa se dio cuenta de las perspectivas que tienen los adolescentes en el ejercicio de su sexualidad, para lo cual se analizaron los discursos escritos de 275 bachilleres de tres comunidades del municipio de Tzimol, Chiapas.

La etapa de la adolescencia es un momento inaugural donde aparecen con mayor intensidad las pulsiones sexuales dirigidas a objetos fuera del ámbito de las relaciones parentales. La ley que se instala en la prohibición del incesto no solo prohíbe la relación sexual con la madre/padre, sino que posibilita la apertura de relaciones sexuales hacia otras mujeres u hombres fuera del núcleo familiar. Esto, sin embargo, se tensa ante el contexto cultural que establece determinados principios, normas y valores socialmente aceptados para el ejercicio de la sexualidad de manera diferenciada para los hombres y para las mujeres. 
Revista Iberoamericana

de las Ciencias Sociales y

Humanísticas

ISSN: $2395-7972$

Las y los jóvenes adolescentes de las comunidades de Tzimol, Chiapas, igual que muchos otros que comparten sus condiciones socioculturales, políticas y económicas en México, se debaten entre la fuerza de la pulsión sexual propia de la edad y las consecuencias desfavorables para sus expectativas de desarrollo personal, de iniciar una vida sexual activa a temprana edad.

Dependiendo de cada género, encontramos argumentos racionales diferentes para postergar el tiempo de inicio de experiencias sexuales asociadas directamente con roles y responsabilidades propias de la adultez. Para los hombres, por ejemplo, iniciar su práctica sexual debe ir de la mano con ser sujeto de derecho legal para ser libre de tomar sus propias decisiones — lo que se consigue con la mayoría de edad (18 años) — , tener un trabajo para cumplir con su rol de proveedor de su familia, tener la capacidad de ser responsable hacia su familia $\mathrm{y}$, en último término, tener una carrea profesional como una opción para conseguir un trabajo y cumplir con sus responsabilidades familiares. Lo anterior quiere decir que las relaciones sexuales se vinculan directamente con tener una pareja, hijos/as y formalizar una familia, ante lo cual la responsabilidad aparece como el principal valor social que los guía.

En el caso de las mujeres, la más destacada diferencia en comparación con los hombres es su expectativa de iniciar su práctica sexual a una edad más tardía (entre 20 a 25 años) que ellos (entre 18 y 19 años). Sus expectativas giran alrededor de tener una carrera profesional para estar preparadas cuando deban enfrentar la posibilidad de quedar embarazadas, del reconocimiento de que un embarazado puede traer riesgos para su propia vida, de su temor por las consecuencias al no saber ser responsable y de tener las habilidades cognitivas necesarias para responsabilizarse por sus actos.

Ante estos resultados, es innegable que cada realidad tiene un sentido y una significación desde las condiciones y situaciones de género construidas socialmente. Queda, por tanto, profundizar en este tipo de intervenciones para generar nuevos análisis y comprensión del proceso de la adolescencia en nuestro tiempo. 
Revista Iberoamericana

de las Ciencias Sociales y

Humanísticas

ISSN: $2395-7972$

\section{Referencias}

Aberasturi, A. y M. Knobel (1987). La adolescencia Normal, Paidós: México.

Consejo Nacional de Evaluación de la Política de Desarrollo Social [Coneval] (2010). Medición de la pobreza 2010. Recuperado de https://www.coneval.org.mx/Medicion/Paginas/Pobreza-2010.aspx

Díaz, A., Sanhueza, R. y Yaksic, N. (2002). Riesgos obstétricos en el embarazo adolescente: estudio comparativo de resultados obstétricos y perinatales con pacientes embarazadas adultas. Revista Chilena de Obstetricia y Ginecología, 67(6), 481-487.

Díaz-Sánchez V. (2003). El embarazo de las adolescentes en México. Gaceta Médica de México, 139(1), 23-28. Recuperado de http://www.medigraphic.com/pdfs/gaceta/gm2003/gms031e.pdf.

Eddy-Ives, L. S. (2014). La identidad del adolescente. Cómo se construye. Revista Adolescere, $\quad 2(2), \quad 14-18 . \quad$ Recuperado de https://www.adolescenciasema.org/usuario/documentos/02-

01\%20Mesa\%20debate\%20-\%20Eddy.pdf.

Estrategia Nacional para la Prevención del Embarazo en Adolescentes [Enapea] (28 de marzo de 2017). Gobierno de la República. Recuperado de https://www.gob.mx/conapo/articulos/estrategia-nacional-para-la-prevencion-delembarazo-en-adolescentes-enapea

Fondo de Población de las Naciones Unidas [UNFPA] (2013). Maternidad en la niñez. Enfrentar el reto del embarazo en adolescentes. Recuperado de: https://www.unfpa.org/sites/default/files/pub-pdf/ES-SWOP2013.pdf

Freud, S. (1916). La vida sexual de los seres humanos. En Obras completas XX. Buenos Aires: Amorrortu,

Instituto Nacional de Estadística Geografía e Informática [Inegi] (2010). Censo de Población y Vivienda. México, Instituto Nacional de Estadística y Geografía. Recuperado de: https://www.inegi.org.mx/programas/ccpv/2010/

Instituto Nacional de Estadística Geografía e Informática [Inegi] (2014). Encuesta Nacional de la Dinámica Demográfica 2014. La anticoncepción: implicaciones en el embarazo adolescente, fecundidad y salud reproductiva en México: Recuperado de: http://www.beta.inegi.org.mx/app/biblioteca/ficha.html?upc $=702825089627 \mid 7028250$ 89627 


\section{Revista Iberoamericana}

de las Ciencias Sociales y

Humanísticas

ISSN: $2395-7972$

Instituto Nacional de Estadística Geografía e Informática [Inegi] (2015). Consulta $\begin{array}{llll}\text { interactiva de de } & \text { decuperado }\end{array}$ http://www.inegi.org.mx/sistemas/olap/Proyectos/bd/continuas/natalidad/nacimientos $. \operatorname{asp} ? \mathrm{~s}=$ est\&c=23699\&proy=nat_nac

Instituto Nacional de Estadística Geografía e Informática [Inegi] (2016). PIB Entidad Federativa Anual. $\quad$ Recuperado de http://www.inegi.org.mx/est/contenidos/proyectos/cn/pibe/default.aspx.

Instituto Nacional de las Mujeres [Inmujeres] (2016a). Agenda ciudadana por la igualdad de género en Tzimol, Chiapas. Tzimol, Chiapas.

Juárez-Bolaños, L., Parra-Vázquez, M. R., Mariaca-Méndez, R. y Díaz-Hernández, B. M. (2011). Modos de vida de los jóvenes en un espacio rural e indígena de México. Estudios Sociales, $\quad$ 19(38). Recuperado de http://www.scielo.org.mx/scielo.php?script=sci_arttext\&pid=S018845572011000200004 .

Lozano Vicente, A. (2014). Teoría de teorías sobre la adolescencia. Última Década, 22(40), 11-36.

Pérez-Serrano G. (coord.) (2001). Modelos de investigación cualitativa en educación social y animación sociocultural. Aplicaciones prácticas. España: Narcea.

Reyes, D. y González Almontes, E. (2014). Elementos teóricos para el análisis del embarazo adolescente. Sexualidad, Salud y Sociedad, (17), 98-123.

Romero, L. (2016). México, primer lugar en la OCDE. Impulsa Medicina programa para prevenir el embarazo adolescente. Gaceta UNAM, 816(4), 4-5.

Secretaría de Educación Pública [SEP] (2012). Embarazo adolescente y madres jóvenes en México: una visión desde el Promajoven. Organización de las Naciones Unidas.

Secretaría de Educación Pública [SEP] (2013). Embarazo adolescente y madres jóvenes en México: una visión desde el Promajoven. México: SEP.

Secretaría de Salud (2002). Prevención del embarazo no planeado en los adolescentes. México: Secretaria de Salud 
Revista Iberoamericana

de las Ciencias Sociales y

Humanísticas

ISSN: 2395 - 7972

Soto-Martínez, O., Franco-Bonal, A., Franco-Bonal A., Silva-Valido J., y VelázquezZúñiga, G. A. (2003). Embarazo en la adolescencia y conocimientos sobre sexualidad. Revista Cubana de Medicina General Integral, 19(6). Recuperado de http://scielo.sld.cu/scielo.php?script=sci_arttext\&pid=S0864-21252003000600002.

Taylor, S. J. y Bogdan, R. (1996). Introducción a los métodos cualitativos de investigación. España: Paidós.

Unicef (2011). Estado mundial de la infancia 2011. La adolescencia Una época de oportunidades. Estados Unidos: Unicef. 


\section{Revista Iberoamericana de las Ciencias Sociales y Humanísticas}

\begin{tabular}{|c|c|}
\hline Rol de Contribución & Autor (es) \\
\hline Conceptualización & Oscar Cruz Pérez (igual) Hildebertha Esteban Silvestre (igual) \\
\hline Metodología & Oscar Cruz Pérez (igual) Hildebertha Esteban Silvestre (igual) \\
\hline Software & NO APLICA \\
\hline Validación & Oscar Cruz Pérez (igual) Hildebertha Esteban Silvestre (igual) \\
\hline Análisis Formal & $\begin{array}{l}\text { Oscar Cruz Pérez (Principal) Hildebertha Esteban Silvestre } \\
\text { (apoyo) Carlos Eduardo Pérez Jiménez (apoyo) } \\
\text { Jesús Ocaña Zúñiga (apoyo) Germán Alejandro García Lara } \\
\text { (apoyo) }\end{array}$ \\
\hline Investigación & $\begin{array}{l}\text { Oscar Cruz Pérez (Principal) Hildebertha Esteban Silvestre } \\
\text { (igual) }\end{array}$ \\
\hline Recursos & $\begin{array}{l}\text { Oscar Cruz Pérez (Principal) Hildebertha Esteban Silvestre } \\
\text { (apoyo) Jesús Ocaña Zúñiga (apoyo) } \\
\text { Germán Alejandro García Lara (apoyo) } \\
\text { Carlos Eduardo Pérez Jiménez (apoyo) }\end{array}$ \\
\hline Curación de datos & $\begin{array}{l}\text { Oscar Cruz Pérez } \\
\text { Hildebertha Esteban Silvestre (apoyo) } \\
\text { Jesús Ocaña Zúñiga(apoyo) } \\
\text { Germán Alejandro García Lara(apoyo) } \\
\text { Carlos Eduardo Pérez Jiménez(apoyo) }\end{array}$ \\
\hline $\begin{array}{l}\text { Escritura - Preparación del } \\
\text { borrador original }\end{array}$ & Oscar Cruz Pérez (igual) Hildebertha Esteban Silvestre(igual) \\
\hline $\begin{array}{l}\text { Escritura - Revisión y } \\
\text { edición }\end{array}$ & $\begin{array}{l}\text { Oscar Cruz Pérez (Principal) } \\
\text { Hildebertha Esteban Silvestre (apoyo) } \\
\text { Carlos Eduardo Pérez Jiménez(apoyo) }\end{array}$ \\
\hline Visualización & $\begin{array}{l}\text { Oscar Cruz Pérez (Principal) } \\
\text { Hildebertha Esteban Silvestre (apoyo) } \\
\text { Jesús Ocaña Zúñiga (apoyo) } \\
\text { Germán Alejandro García Lara (apoyo) }\end{array}$ \\
\hline Supervisión & $\begin{array}{l}\text { Hildebertha Esteban Silvestre (igual) } \\
\text { Oscar Cruz Pérez (igual) }\end{array}$ \\
\hline $\begin{array}{l}\text { Administración de } \\
\text { Proyectos }\end{array}$ & Oscar Cruz Pérez \\
\hline Adquisición de fondos & Oscar Cruz Pérez \\
\hline
\end{tabular}

